\title{
Dynamic stress intensity factor of a functionally graded material under antiplane shear loading
}

\author{
C. Li and G. J. Weng, New Brunswick, New Jersey, Z. Duan, Beijing, and \\ Z. Zou, Shijiazhuang, China
}

(Received March 1, 2000; revised April 10, 2000)

\begin{abstract}
Summary. The dynamic response of a finite crack in an unbounded Functionally Graded Material (FGM) subjected to an antiplane shear loading is studied in this paper. The variation of the shear modulus of the functionally graded material is modeled by a quadratic increase along the direction perpendicular to the crack surface. The dynamic stress intensity factor is extracted from the asymptotic expansion of the stresses around the crack tip in the Laplace transform plane and obtained in the time domain by a numerical Laplace inversion technique. The influence of graded material property on the dynamic intensity factor is investigated. It is observed that the magnitude of dynamic stress intensity factor for a finite crack in such a functionally graded material is less than in the homogeneous material with a property identical to that of the FGM crack plane.
\end{abstract}

\section{Introduction}

In recent years the concept of Functionally Graded Materials (FGMs) has been introduced to the development of structural components. The advantages of FGMs are that the materials can resist corrosion, radiation and high temperatures more effectively and, at the same time, the residual and thermal stresses in the materials can be relaxed significantly. Due to these advantages, the interests in FGM research are growing rapidly. It is obvious that FGMs are becoming an important area in the development of advanced composite materials.

For many engineering applications the components made of FGMs could be subjected to time-dependent loadings. The graded material properties of the FGM in addition to the presence of mechanical imperfections, such as cracks, can have a strong influence on the dynamic response of these components. A good knowledge of the dynamic response of this kind of components is essential to achieving an in depth understanding of the failure mechanisms of the FGM. Although significant attention has been paid to various theoretical and practical aspects of the mechanical behavior of FGMs and there has also been a considerable bulk of studies on the fracture of FGMs [1], reports on their dynamic fracture mechanics are still very few. Among this limited studies, Nakagaki and his co-workers [2] made a numerical treatment of fracture occurring in an FGM under dynamic load. Parameswaran and Shukla [3] studied dynamic fracture in FGMs having discrete property variations using dynamic photoelasticity. Babaei and Lukasiewicz [4] investigated the dynamic response of a crack in an FGM between two dissimilar half planes under antiplane shear impact by using dual integral equations. An exponential function for the variation of material properties was used in their article. Wang and his co-workers [5] considered the dynamic fracture mechanics analysis for a composite 
material with a material inhomogeneity in the thickness direction. They used the model of stacked homogeneous layers and the singular integral equation technique. Li and Zou [6] reported the torsional impact response of an FGM with a penny-shaped crack by using dual integral equations.

In this paper, we present the antiplane impact response of an unbounded FGM with a Griffith crack. The main objective is to investigate the effect of material inhomogeneity on the dynamic response of FGMs with cracks. By using Laplace transforms for the time variable and Fourier transform for the space variable, the problem is reduced to a pair of dual integral equations. Following the procedure of Copson [7], the solution of the dual integral equations is expressed with a Fredholm integral equation of the second kind. The dynamic stress intensity factor is extracted from the asymptotic expansion of the stresses around the crack tip in the Laplace transformed plane. The dynamic stress intensity factor in the time domain is obtained by the Laplace numerical inversion technique. The influence of material inhomogeneity on the dynamic stress intensity factor is demonstrated graphically.

\section{Formulation of the problem}

Consider an unbounded functionally graded material to which a rectangular $x y z$-coordinates system is attached, as shown in Fig. 1. Assume a finite crack of length $2 a$ is situated in the $y=0$ plane and subjected to an antiplane shear impact. Let the components of the displacement in the $x, y$ and $z$ directions be labeled by $u_{x}, u_{y}$ and $u_{z}$, respectively. For antiplane shear motion, $u_{x}$ and $u_{y}$ vanish everywhere and $u_{z}$ is a function of $x, y$ and $t$, i.e.,

$u_{x}=u_{y}=0, \quad u_{z}=w(x, y, t)$

where $t$ is time. Assuming that the material is isotropic, the nonvanishing stress components $\tau_{x z}$ and $\tau_{y z}$ are as follows:

$\tau_{x z}=\mu(y) \frac{\partial w}{\partial x}, \quad \tau_{y z}=\mu(y) \frac{\partial w}{\partial y}$,

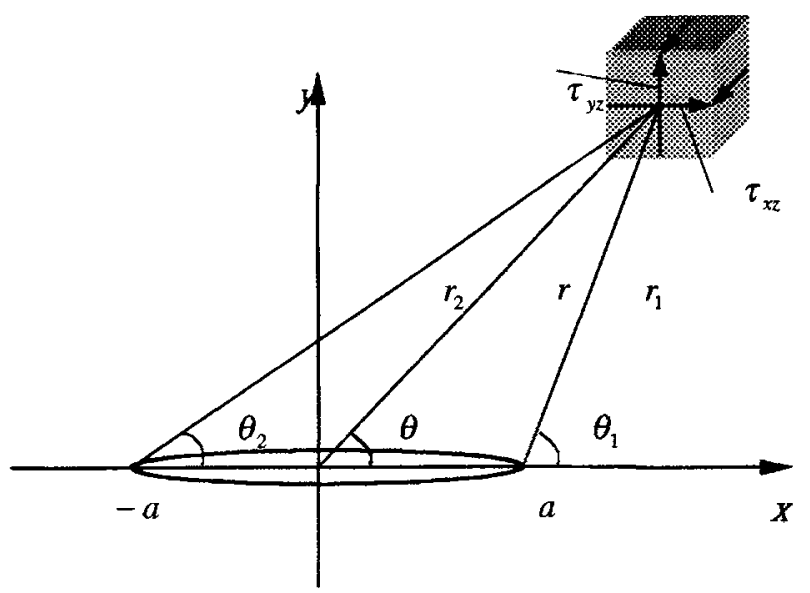

Fig. 1. Crack geometry and stress components 
where $\mu(y)$ is the position-dependent shear modulus. The equation of motion can be written as

$\frac{\partial^{2} w}{\partial x^{2}}+\frac{\partial^{2} w}{\partial y^{2}}+\frac{\mu^{\prime}(y)}{\mu(y)} \frac{\partial w}{\partial y}=\frac{\varrho(y)}{\mu(y)} \frac{\partial^{2} w}{\partial t^{2}}$,

where $\mu^{\prime}(y)$ is the derivative of $\mu(y)$, and $\varrho(y)$ is the mass density of the FGM.

Suppose that the material is initially at rest. At time $t=0$, an antiplane shear stress of magnitude $\tau_{0}$ is suddenly applied to the crack surfaces and maintained at the same constant value thereafter. Hence, the boundary conditions are given as follows:

$\tau_{y z}(x, 0, t)=-\tau_{0} H(t), \quad 0 \leq|x|<a ; t>0$,

$w(x, 0, t)=0, \quad|x| \geq a ; t>0$,

where $H(t)$ is the Heaviside unit step function. The initial conditions are zero.

\section{Material property models}

There are two material parameters involved in this problem: the shear modulus $\mu$ and the density $\varrho$. Due to the mathematical complexity it is necessary to assume that the shear modulus and the mass density can vary independently. Such an idealization can offer considerable simplifications to the analysis. In static problems, several models have been proposed for describing the variation of $\mu$. One of them is a power function,

$\mu(y)=\mu_{0}|y|^{m}, \quad 0 \leq m<1$.

This model was used by Sih and Chen [8], but it has an undesirable feature that at $y=0$ the shear modulus becomes zero. The second model is an exponential form of the type

$\mu(y)=\mu_{0} \exp (\alpha y)$.

This model was extensively used by Erdogan and his co-workers for inhomogeneous elastic materials with cracks subjected to mechanical loadings [9]-[11], and by Noda and his coworkers for crack problems in inhomogeneous thermoelastic solids under thermal loadings [12], [13]. The third model is a linear function

$\mu(y)=\mu_{0}(1+c|y|)$,

proposed by Gerasoulis and Srivastav [14], and the fourth model is

$\mu(y)=(a+b y)^{k}$,

proposed by Wang and his co-workers [15] for studying the problems of an interface crack in composites with an inhomogeneous interlayer.

However, in order to obtain the dynamic response of an inhomogeneous FGM, most of the models will present considerable difficulties due to the presence of the inertia term. We have found that the variation

$\mu(y)=\mu_{0}(1+\alpha|y|)^{2}, \alpha>0 ; \quad \varrho=$ constant

is mathematically tractable, and still physically representative enough. In the above equations, $\mu_{0}$ is the value at $y=0$. 
We realized that, by taking $\varrho$ as constant, it is an idealization, but in some practical systems, such as $\mathrm{TiC} / \mathrm{Ti}$ or $\mathrm{TiB}_{2} / \mathrm{Ti}$, the variation of the mass density is almost constant. In any event, we find that with this model several interesting features of the dynamic fracture problem in FGMs can be brought to light.

\section{Derivation of integral equation}

The standard Laplace transform on $f(t)$ is

$f^{*}(p)=\int_{0}^{\infty} f(t) e^{-p t} d t$

whose inversion is

$f(t)=\frac{1}{2 \pi i} \int_{\mathrm{Br}} f^{*}(p) e^{p t} d p$,

where $\mathrm{Br}$ denotes the Bromwich path of integration, which is a line on the right-hand side of the $p$-plane and parallel to the imaginary axis. Applying Eq. (11) to Eq. (3) and noting that the mass density is now a constant, we obtain the transformed equation

$\frac{\partial^{2} w^{*}}{\partial x^{2}}+\frac{\partial^{2} w^{*}}{\partial y^{2}}+\frac{\mu^{\prime}(y)}{\mu(y)} \frac{\partial w^{*}}{\partial y}=\frac{\varrho p^{2}}{\mu(y)} w^{*}$.

Considering the symmetry of the problem about $x$ and $y$, we only need to consider the first quadrant of the $x-y$ plane. Introducing the pair of Fourier cosine transforms

$f^{c}(s)=\int_{0}^{\infty} f(x) \cos (s x) d x$,
$f(x)=\frac{2}{\pi} \int_{0}^{\infty} f^{c}(s) \cos (s x) d s$

and defining

$w^{*}(x, y, p)=\frac{2}{\pi} \int_{0}^{\infty} U(s, y, p) \cos (s x) d s$,

then Eq. (13) can be transformed into

$\frac{\partial^{2} U(s, y, p)}{\partial y^{2}}+\frac{\mu^{\prime}(y)}{\mu(y)} \frac{\partial U(s, y, p)}{\partial y}-\left[s^{2}+\frac{\varrho p^{2}}{\mu(y)}\right] U(s, y, p)=0$.

Substituting (10) into (17), we obtain

$\frac{\partial^{2} U(s, y, p)}{\partial y^{2}}+\frac{2 \alpha}{1+\alpha y} \frac{\partial U(s, y, p)}{\partial y}-\left[s^{2}+\frac{\varrho p^{2}}{\mu_{0}(1+\alpha y)^{2}}\right] U(s, y, p)=0$.

By defining

$X=s(1+\alpha y), \quad Y=(1+\alpha y)^{1 / 2} U$, 
Eq. (18) can be rewritten as

$\frac{d^{2} Y}{d X^{2}}+\frac{1}{X} \frac{d Y}{d X}-\left[\frac{1}{\alpha^{2}}+\frac{\beta^{2}}{X^{2}}\right] Y=0$

where

$\beta=\sqrt{\frac{1}{4}+\frac{\varrho p^{2}}{\mu_{0} \alpha^{2}}}$

It can be seen that Eq. (20) is a standard modified Bessel differential equation.

From the solution of the modified Bessel Eq. (20) and considering the regularity condition at $y \rightarrow \infty$, the solution of Eq. (18) can be expressed as

$U(s, y, p)=A(s, p)(1+\alpha y)^{-1 / 2} K_{\beta}\left[(1+\alpha y) \frac{s}{\alpha}\right]$,

where $K_{\beta}()$ is the modified Bessel function of the second kind.

Substituting Eq. (22) into Eq. (16), we obtain

$w^{*}(x, y, p)=\frac{2}{\pi} \int_{0}^{\infty} A(s, p)(1+\alpha y)^{-1 / 2} K_{\beta}\left[(1+\alpha y) \frac{s}{\alpha}\right] \cos (s x) d s$

Then from the Laplace transformations of the stresses $\tau_{y z}$ and $\tau_{x z}$, we obtain

$$
\begin{aligned}
\tau_{y z}^{*}(x, y, p)= & \mu(y) \frac{2}{\pi} \int_{0}^{\infty} A(s, p)\left\{-\frac{\alpha}{2}(1+\alpha y)^{-3 / 2} K_{\beta}\left[(1+\alpha y) \frac{s}{\alpha}\right]\right. \\
& \left.+s(1+\alpha y)^{-1 / 2} K_{\beta}{ }^{\prime}\left[(1+\alpha y) \frac{s}{\alpha}\right]\right\} \cos (s x) d s \\
\tau_{x z}^{*}(x, y, p)= & -\mu(y) \frac{2}{\pi} \int_{0}^{\infty} A(s, p)(1+\alpha y)^{1 / 2} K_{\beta}\left[(1+\alpha y) \frac{s}{\alpha}\right] \sin (s x) s d s
\end{aligned}
$$

where $K_{\beta}{ }^{\prime}()$ is the derivative of $K_{\beta}()$ with respect to $y$.

In the Laplace transform domain, the conditions on the plane $y=0$ become

$\tau_{y z}^{*}(x, 0, p)=-\frac{\tau_{0}}{p}, \quad 0 \leq x<a$

$w^{*}(x, 0, p)=0, \quad x \geq a$.

From Eqs. (23), (24), (26) and (27), a pair of dual integral equations is obtained as

$$
\begin{aligned}
& \int_{0}^{\infty} B(s, p) \cos (s x) d s=0, \quad x \geq a, \\
& \int_{0}^{\infty} s B(s, p) G(s, p) \cos (s x) d s=\frac{\pi \tau_{0}}{2 \mu_{0} p}, \quad 0 \leq x<a,
\end{aligned}
$$


where

$$
\begin{aligned}
B(s, p) & =A(s, p) K_{\beta}\left(\frac{s}{\alpha}\right), \\
G(s, p) & =\frac{\frac{\alpha}{2} K_{\beta}\left(\frac{s}{\alpha}\right)-s K_{\beta}^{\prime}\left(\frac{s}{\alpha}\right)}{s K_{\beta}\left(\frac{s}{\alpha}\right)} .
\end{aligned}
$$

The dual integral Eqs. (28) and (29) can be solved by applying the method of Copson [7]. It is found as following:

$B(s, p)=\frac{\pi \tau_{0} a^{2}}{2 \mu_{0} p} \int_{0}^{1} \sqrt{\xi} \Phi^{*}(\xi, p) J_{0}(s a \xi) d \xi$,

where $J_{0}$ is the zeroth-order Bessel function of the first kind and the function $\Phi^{*}(\xi, p)$ is governed by a Fredholm integral equation of the second kind,

$\Phi^{*}(\xi, p)+\int_{0}^{1} \Phi^{*}(\xi, p) \mathrm{M}(\xi, \eta, p) d \eta=\sqrt{\xi}$.

The kernel function $\mathrm{M}(\xi, \eta, p)$ in Eq. (33) is

$\mathrm{M}(\xi, \eta, p)=\sqrt{\xi \eta} \int_{0}^{\infty} s\left[G\left(\frac{s}{a}, p\right)-1\right] J_{0}(s \xi) J_{0}(s \eta) d s$.

\section{Dynamic stress intensity factor}

As in the static case, the amplitude of the stress field around the crack tip in the dynamic situation is measured by a stress intensity factor, which varies as a function of time. The Laplace transform of the dynamic stress intensity factor can be extracted from the asymptotic expansion of the stresses around the crack tip in the Laplace transform domain.

Let $B(s, p)$ be expressed as

$B(s, p)=\frac{\pi \tau_{0} a}{2 \mu_{0} p} \frac{1}{s}\left\{\Phi^{*}(1, p) J_{1}(s a)-\int_{0}^{1} \xi J_{1}(s a \xi) \frac{d}{d \xi}\left[\xi^{-1 / 2} \Phi^{*}(\xi, p)\right] d \xi\right\}$.

From Eqs. (35), (30), (24) and (25) and considering that when $s \rightarrow \infty$, which is corresponding to the crack tip in the Laplace domain, we have the following asymptotic behavior of $K_{\beta}(s)$ and $K_{\beta}{ }^{\prime}(s)$ :

$$
\begin{aligned}
& K_{\beta}(s)=\sqrt{\frac{\pi}{2 s}} e^{-s}\left[1+O\left(\frac{1}{s}\right)\right], \\
& K_{\beta}^{\prime}(s)=-\sqrt{\frac{\pi}{2 s}} e^{-s}\left[1+O\left(\frac{1}{s}\right)\right] .
\end{aligned}
$$


Consequently we obtain

$$
\begin{aligned}
\tau_{y z}^{*}(x, y, p) & =\frac{\tau_{0} a \mu(y)}{\mu_{0}} \frac{\Phi^{*}(1, p)}{p} \int_{0}^{\infty} \frac{(1+\alpha y)^{-1 / 2} K_{\beta}{ }^{\prime}\left[(1+\alpha y) \frac{s}{\alpha}\right]}{K_{\beta}\left(\frac{s}{\alpha}\right)} J_{1}(s a) \cos (s x) d s+\ldots \\
& =-\frac{\tau_{0} a \mu(y)}{\mu_{0}} \frac{\Phi^{*}(1, p)}{p}(1+\alpha y)^{-1} \int_{0}^{\infty} J_{1}(s a) \exp (-s y) \cos (s x) d s+\ldots \\
\tau_{x z}^{*}(x, y, p) & =\frac{\tau_{0} a \mu(y)}{\mu_{0}} \frac{\Phi^{*}(1, p)}{p} \int_{0}^{\infty} \frac{(1+\alpha y)^{-1 / 2} K_{\beta}\left[(1+\alpha y) \frac{s}{\alpha}\right]}{K_{\beta}\left(\frac{s}{\alpha}\right)} J_{1}(s a) \sin (s x) d s+\ldots \\
& =-\frac{\tau_{0} a \mu(y)}{\mu_{0}} \frac{\Phi^{*}(1, p)}{p}(1+\alpha y)^{-1} \int_{0}^{\infty} J_{1}(s a) \exp (-s y) \sin (s x) d s+\ldots
\end{aligned}
$$

Evaluation of the integrals in Eqs. (38) and (39) yields

$$
\begin{aligned}
& \int_{0}^{\infty} J_{1}(s a) \exp (-s y) \cos (s x) d s=\frac{1}{a}-\frac{1}{\sqrt{r_{1} r_{2}}} \frac{r}{a} \cos \left(\theta-\frac{1}{2} \theta_{1}-\frac{1}{2} \theta_{2}\right), \\
& \int_{0}^{\infty} J_{1}(s a) \exp (-s y) \sin (s x) d s=-\frac{1}{\sqrt{r_{1} r_{2}}} \frac{r}{a} \sin \left(\theta-\frac{1}{2} \theta_{1}-\frac{1}{2} \theta_{2}\right) .
\end{aligned}
$$

The polar coordinates $r, r_{1}, r_{2}, \theta, \theta_{1}$ and $\theta_{2}$ are defined in Fig. 1.

Substituting Eqs. (40), (41) and (10) into Eqs. (38) and (39) and letting $r \rightarrow a, \theta \rightarrow 0$, $\theta_{2} \rightarrow 0$ and $r_{2} \rightarrow 2 a$, we obtain the local stress field

$$
\begin{aligned}
& \tau_{y z}^{*}\left(r_{1}, \theta_{1}, p\right)=\frac{K_{I I}^{*}(p)}{\sqrt{2 \pi r_{1}}} \cos \left(\frac{\theta_{1}}{2}\right)+O\left(r_{1}^{0}\right), \\
& \tau_{x z}^{*}\left(r_{1}, \theta_{1}, p\right)=\frac{K_{I I I}^{*}(p)}{\sqrt{2 \pi r_{1}}} \sin \left(\frac{\theta_{1}}{2}\right)+O\left(r_{1}^{0}\right) .
\end{aligned}
$$

The Laplace transform of the dynamic stress intensity factor $K_{I I I}^{*}(p)$ in Eqs. (42) and (43) is

$$
K_{I I I}^{*}(p)=\tau_{0} \sqrt{\pi a} \frac{\Phi^{*}(1, p)}{p}
$$

where $\Phi^{*}(1, p)$ is the value of $\Phi^{*}(\xi, p)$ evaluated at the crack tip corresponding to $\xi=1$.

The dynamic stress intensity factor in the time domain can be obtained by

$$
K_{I I I}(t)=\tau_{0} \sqrt{\pi a} \frac{1}{2 \pi i} \int_{\mathrm{Br}} \frac{\Phi^{*}(1, p)}{p} e^{p t} d p .
$$



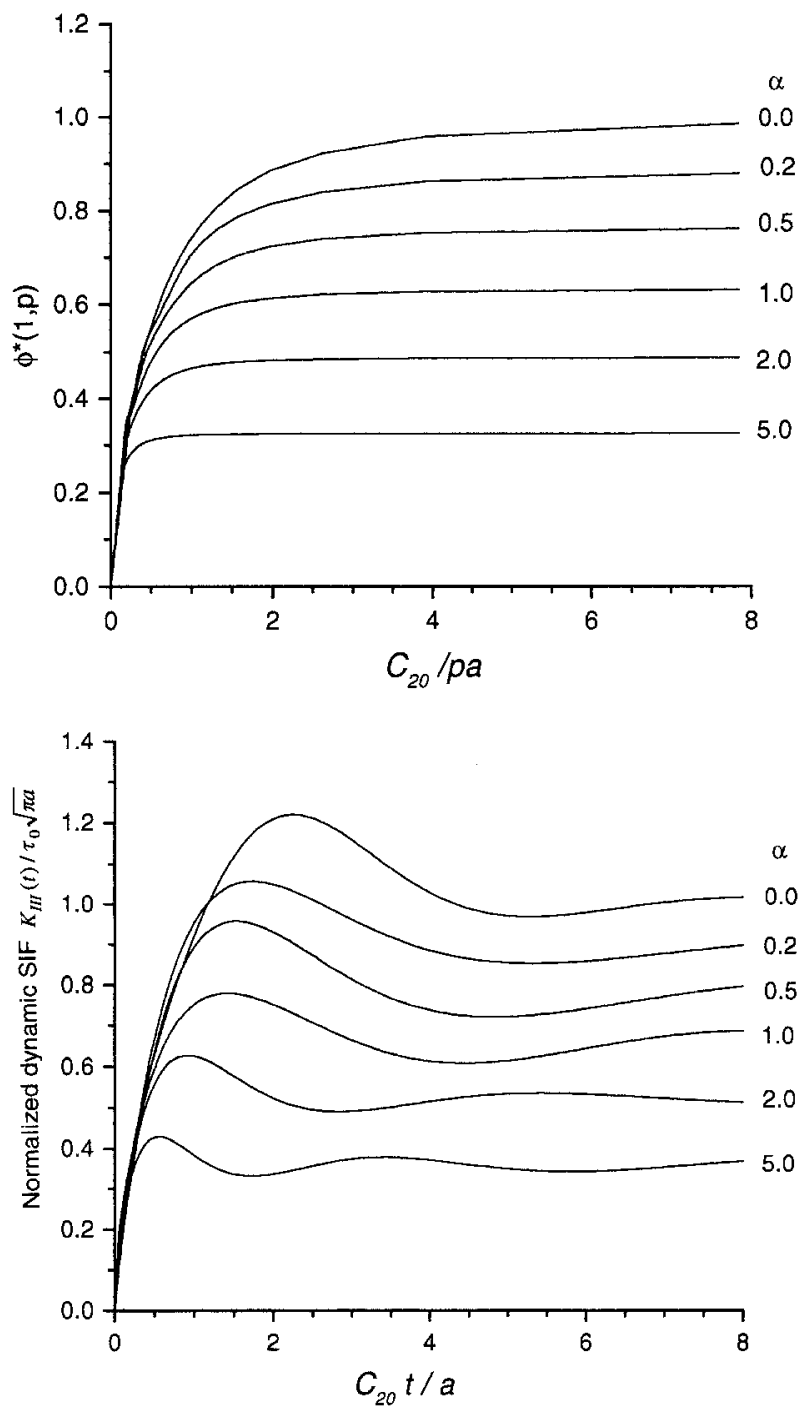

Fig. 2. Variation of $\Phi^{*}(1, p)$ with $C_{20} / p a$
Fig. 3. Normalized dynamic stress intensity factor $K_{I I I}(t) / \tau_{0} \sqrt{\pi a}$ versus time

\section{Results and discussion}

The functional dependence of the stresses on $r_{1}$ and $\theta_{1}$ as shown in Eqs. (42) and (43) reveals that the dynamic stresses in functionally graded materials with cracks also possess the inverse square root singularity in terms of $r_{1}$ and that the angular distribution in $\theta_{1}$ is the same as in the case of homogeneous solids with cracks.

Equation (45) shows that the expressional form of the dynamic stress intensity factor for functionally graded materials is identical to that for homogeneous materials. However, the value of the dynamic stress intensity factor for a crack in a functionally graded material is different from that for a crack in a homogeneous material.

By using the numerical Laplace transform inversion scheme described by Miller and Guy [16], the dynamic stress intensity factor expressed by Eq. (45) can be evaluated. It is apparent, however, that $\Phi^{*}(1, p)$ must be known first before the evaluation of Eq. (45) can be performed. Figure 2 shows the numerical results of $\Phi^{*}(1, p)$ as functions of the dimensionless Laplace transform wave number $C_{20} / p a$ for several different values of $\alpha$. Here $C_{20}=\sqrt{\mu / \varrho}$. 
The influence of the inhomogeneity parameter $\alpha$ on $\Phi^{*}(1, p)$ can be seen clearly: the magnitude of $\Phi^{*}(1, p)$ decrease as $\alpha$ increases.

The influence of $\alpha$ on the dynamic stress intensity factor $K_{I I I}(t)$ is displayed in Fig. 3, where the normalized $K_{I I I}(t) / \tau_{0} \sqrt{\pi a}$ is plotted as a function of $C_{20} t / a$. It is observed that all the curves reach a peak and then oscillate about the static values. As $\alpha$ - or the material inhomogeneity - increase, the SIF also decreases. Since the condition $\alpha=0$ corresponds to a homogeneous material whose properties are equal to that of the FGM at the crack surface, the peak values of the dynamic stress intensity factor for FGMs are less than that for the homogeneous material. This has the strong implication that the crack driving force can be reduced by using FGMs to replace homogeneous materials in engineering structures. It is also revealed that the time required to reach the peak SIF decreases with increasing $\alpha$.

\section{Conclusions}

In this paper, the transient response of FGMs with a finite crack under antiplane shear impact is studied. The local stress field around the crack tip is found to be similar to that for a homogeneous material. This result is very important in that one can use conventional numerical methods of dynamic fracture mechanics developed for homogeneous solids, such as the dynamic fracture finite element method, to analyze the dynamic crack problems in FGMs.

The computed dynamic stress intensity factors show that the graded material property of an FGM has a considerable influence on the fracture behavior of FGMs with cracks. It can be concluded that under the FGM property model proposed in this paper, i.e., the shear modulus increases symmetrically along the direction perpendicular to the crack surface and the mass density keeps constant, the peak value of the dynamic stress intensity factor decreases with the increasing gradient of FGMs. Furthermore, the greater the gradient of an FGM, the faster the dynamic stress intensity factor reaches the peak.

\section{Acknowledgement}

The work of C. Li was supported by the China Scholarship Council and the National Science Foundation of USA under CMS-9625304. G. J. Weng was supported by the National Science Foundation of USA, Mechanics and Material Program, under CMS-9625304. Z. Duan was supported by the National Natural Science Fund of China under the Key Project No. 19891180. Z. Zou was supported by the National Natural Science Fund of China under the project No. 19772029.

\section{References}

[1] Erdogan, F.: Fracture mechanics of functionally graded materials. Comp. Engng. 5, 753-770 (1995).

[2] Nakagaki, M., Sasaki, H., Hagihara S.: A study of crack in functionally graded material under dynamic loading. Dynamic fracture failure and deformation. ASME PVP 300, 1-6 (1995).

[3] Parameswaran, V., Shukla, A.: Dynamic fracture of a functionally gradient material having discrete property variation. J. Mater. Sci. 33, 3303-3311 (1998).

[4] Babaei, R., Lukasiewicz, S. A.: Dynamic response of a crack in a functionally graded material between two dissimilar half planes under antiplane shear impact load. Engng. Fract. Mech. 60, $479-487$ (1998). 
[5] Wang, B. L., Han, J. C., Du, S. Y.: Dynamic fracture mechanics analysis for composite materials with material inhomogeneity in thickness direction. Acta Mech. Solida Sinica 11, 84-93 (1998).

[6] Li, C., Zou, Z.: Torsional impact response of a functionally graded material with a penny-shaped crack. J. Appl. Mech. 66, 566-567 (1999).

[7] Copson, E. T.: On certain dual integral equations. Proc. Glasgow Math. Asso. 5, 19-24 (1961).

[8] Sih, G. C., Chen, E. P.: Mechanics of fracture 6: Cracks in composite materials. The Hague: Martinus Nijhoff 1981.

[9] Delale, F., Erdogan, F.: The crack problem for a nonhomogeneous plane. J. Appl. Mech. 50, $609-614$ (1983).

[10] Konda, N., Erdogan, F.: The mixed mode crack problem in a nonhomogeneous elastic medium. Engng. Fract. Mech. 47, 533-545 (1994).

[11] Erdogan, F., Wu, B. H.: The surface crack problem for a plate with functionally graded properties. J. Appl. Mech. 64, $449-456$ (1997).

[12] Noda, N., Jin, Z.-H.: Thermal stress intensity factors for a crack in a strip of a functionally gradient material. Int. J. Solids Struct. 30, 1039-1056 (1993).

[13] Jin, Z.-H., Noda, N.: Transient thermal stress intensity factors for a crack in a semi-infinte plate of a functionally gradient material. Int. J. Solids Struct. 31, $203-218$ (1994).

[14] Gerasoulis, A., Srivastav, R. P.: A Griffith crack problem for a nonhomogeneous medium. Int. J. Engng Sci. 18, $239-247$ (1980).

[15] Wang, X. Y., Zou, Z. Z., Wang, D.: On the Griffith crack in a nonhomogeneous interlayer of adjoining two different elastic materials. Int. J. Fract. 79, R51-R56 (1996).

[16] Miller, M. K., Guy, W. T.: Numerical inversion of the Laplace transform by use of Jacobi polynomials. SIAM J. Numer. Analysis 3, 624-635 (1966).

Authors' addresses: Dr. C. Li and Prof. G. J. Weng, Department of Mechanical and Aerospace Engineering, Rutgers University, New Brunswick, NJ 08903, USA (E-mail: weng@jove.rutgers.edu); Dr. C. Li is now a postdoctoral fellow in Department of Mechanical Engineering, University of Delaware, DE 19716, USA; Prof. Z. Duan, LNM, Institute of Mechanics, Chinese Academy of Sciences, Beijing, 100080, China; Prof. Z. Zou, Department of Communications Engineering, Shijiazhuang Railway Institute, Shijiazhuang, 050043, China 